\title{
Synthesis and Antibacterial Screening of Some 1-Aroyl-3-aryl Thiourea Derivatives
}

\author{
Love Kumar Soni, Tamanna Narsinghani, and Rica Jain \\ School of Pharmacy, Devi Ahilya University, Ring Road, Indore 452017, India \\ Correspondence should be addressed to Love Kumar Soni; lovesoni@hotmail.com
}

Received 15 October 2013; Accepted 17 December 2013; Published 29 January 2014

Academic Editors: A. Contini, F. A. Pasha, and Y. S. Prabhakar

Copyright (C) 2014 Love Kumar Soni et al. This is an open access article distributed under the Creative Commons Attribution License, which permits unrestricted use, distribution, and reproduction in any medium, provided the original work is properly cited.

\begin{abstract}
A series of 1-aroyl-3-aryl thioureas derivatives were synthesized and evaluated for antibacterial activity. The results indicated that the compounds possessed higher activity against gram-negative bacteria than gram-positive bacteria. Amongst all these compounds, C18 (89.4\%) exhibited the greatest antibacterial activity against gram-negative bacteria while C5 (85.6\%) displayed maximum antibacterial activity against gram-positive bacteria. Preliminary study of the structure-activity relationship revealed that an electronic factor on aryl rings had a great effect on the antibacterial activity of these compounds.
\end{abstract}

\section{Introduction}

The increased use of antibacterial and antifungal agents has resulted in the development of drug resistant microbial pathogens which results in failure in clinical application of these agents. This highlights the incessant need for the development of new classes of antimicrobial agents and alteration of known drugs in such way that would allow them to retain their physiological action but reduce their resistance to the pathogen. Thiourea derivatives are versatile building blocks for the synthesis of a variety of heterocyclic compounds and display a wide spectrum of biological activities such as antimicrobial [1-4], antimalarial [5], antitubercular [6], anticancer [7], anti-HIV [8], carbonic anhydrases inhibitor [9], appetite suppressants [10], $\beta 3$-adrenergic receptor agonists [11], and CNS activity [12]. Therefore, their beneficial properties have prompted several groups to study these compounds. We have been interested in antimicrobial agents for a few years and have performed QSAR analysis and synthesis of antimicrobial agents [13-15]. Here in the present work we report the synthesis and screening of some 1-aroyl-3-aryl thiourea derivatives for their antimicrobial potency.

\section{Experimental}

Synthesis of some 1-aroyl-3-aryl thioureas (C1-C18) (Figure 1 and Table 1) was carried out by treating substituted benzoyl chloride with an equimolar quantity of potassium thiocyanate in acetone followed by reaction with an equimolar amount of substituted anilines to furnish the 1-aroyl-3-aryl thiourea derivatives.

2.1. General Procedure for the Synthesis of 1-Aroyl-3-aryl Thioureas. A solution of substituted benzoyl chloride $(10 \mathrm{mmol})$ in acetone $(50 \mathrm{~mL})$ was added dropwise to a suspension of potassium thiocyanate $(10 \mathrm{mmol})$ in acetone $(30 \mathrm{~mL})$ and the reaction mixture was refluxed for $30 \mathrm{~min}$. After cooling to room temperature, a solution of substituted aniline $(10 \mathrm{mmol})$ in acetone $(10 \mathrm{~mL})$ was added and the resulting mixture refluxed for $2-3 \mathrm{~h}$. The reaction was monitored by performing TLC using petroleum ether: ethyl acetate $(8: 2)$ as mobile phase. The reaction mixture was poured into cold water and the precipitated thioureas were recrystallized using aqueous ethanol [3].

The structures were confirmed by spectroscopic data and elemental analyses. All the synthesized compounds were 

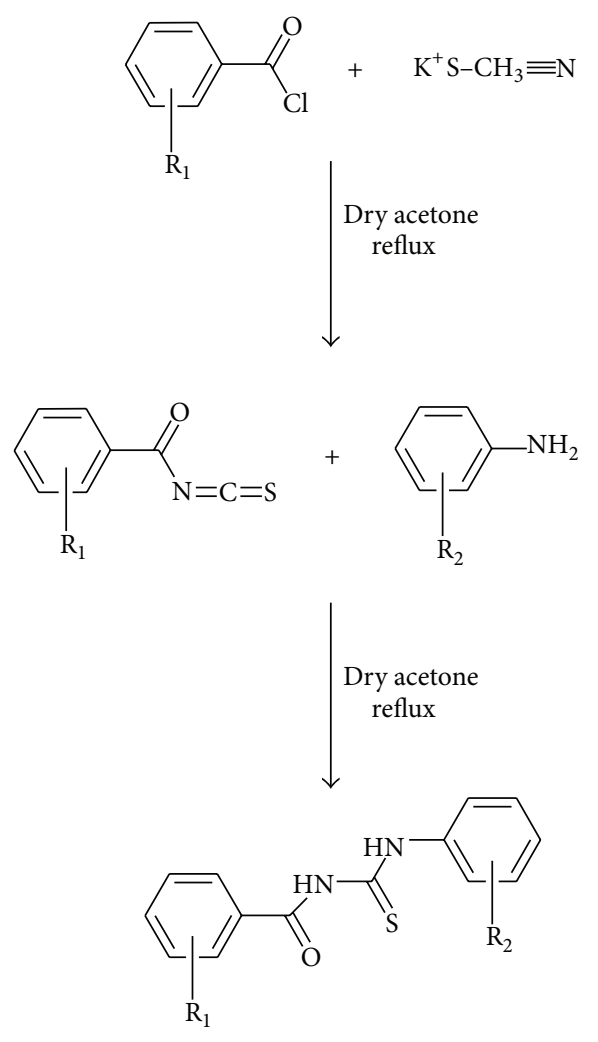

Figure 1: Synthesis of 1-aroyl-3-aryl thiourea.

assayed in vitro for their antibacterial activity. The antibacterial activity of compounds was determined by the paper disc diffusion method using Mueller-Hinton agar medium. Sodium Penicillin G was used as the reference antibacterial agent.

1-(2-Chlorobenzoyl)-3-(4-bromophenyl) Thiourea (C1). Yield $69 \%, R_{f}=0.44$, M.P. $160-165^{\circ} \mathrm{C}$, IR $(\mathrm{KBr}) v\left(\mathrm{~cm}^{-1}\right): 1539.09$ $(\mathrm{C}=\mathrm{C}), 1676.99(\mathrm{C}=\mathrm{O}), 3246.94(\mathrm{~N}-\mathrm{H}), 1159.14(\mathrm{C}=\mathrm{S}), 1159.14$ $(\mathrm{C}-\mathrm{N}), 814.87(\mathrm{C}-\mathrm{Cl}) .{ }^{1} \mathrm{H}$ NMR $\left(\mathrm{CDCl}_{3}\right) \delta: 12.37(1 \mathrm{H}, \mathrm{s}, \mathrm{NH})$, $9.276(1 \mathrm{H}, \mathrm{s}, \mathrm{NH}), 7.43-7.66(\mathrm{~m}, 4 \mathrm{H}$, aroyl ring), 7.26-7.4 (m, $4 \mathrm{H}$, aryl ring). DART-MS m/e (\%): 369. Elemental analysis for $\mathrm{C}_{14} \mathrm{H}_{10} \mathrm{~N}_{2} \mathrm{OSClBr}(\mathrm{MW}=369.66)$ in wt $\%$ calc. $\mathrm{C}=45.48$, $\mathrm{H}=2.73, \mathrm{~N}=7.57, \mathrm{O}=4.33, \mathrm{~S}=8.67$ and found to be $\mathrm{C}=46.01, \mathrm{H}=3.04, \mathrm{~N}=7.42, \mathrm{O}=6.24, \mathrm{~S}=8.16$.

1-(4-Chlorobenzoyl)-3-(3-methoxyphenyl) Thiourea (C2). Yield $62 \%, R_{f}=0.42$, M.P. $130-135^{\circ} \mathrm{C}$, IR (KBr) v $\left(\mathrm{cm}^{-1}\right): 1532.34$ $(\mathrm{C}=\mathrm{C}), 1672.17(\mathrm{C}=\mathrm{O}), 3295(\mathrm{~N}-\mathrm{H}), 1150.46(\mathrm{C}=\mathrm{S}), 1276.79$ $(\mathrm{C}-\mathrm{N}), 850.55(\mathrm{C}-\mathrm{Cl}) .{ }^{1} \mathrm{H} \mathrm{NMR}\left(\mathrm{CDCl}_{3}\right) \delta: 12.52(1 \mathrm{H}, \mathrm{s}, \mathrm{NH})$, $9.03(1 \mathrm{H}, \mathrm{s}, \mathrm{NH}), 7.47-7.85(\mathrm{~m}, 4 \mathrm{H}$, aroyl ring), 6.81-7.2 (m, $4 \mathrm{H}$, aryl ring), 3.83 (3H, s, $\mathrm{OCH}_{3}$ ). DART-MS m/e (\%): 319.07. Elemental analysis for $\mathrm{C}_{15} \mathrm{H}_{13} \mathrm{~N}_{2} \mathrm{O}_{2} \mathrm{SCl}(\mathrm{MW}=320.79)$ in wt $\%$ calc. $\mathrm{C}=56.16, \mathrm{H}=4.08, \mathrm{~N}=8.73, \mathrm{O}=9.97, \mathrm{~S}=9.99$ and found to be $\mathrm{C}=56.12, \mathrm{H}=4.27, \mathrm{~N}=8.59, \mathrm{O}=11.34$, $\mathrm{S}=9.56$.

1-(4-Methoxybenzoyl)-3-(3-methoxyphenyl) Thiourea (C3). Yield $61 \%, R_{f}=0.3$, M.P. $80-85^{\circ} \mathrm{C}$, IR $(\mathrm{KBr}) v\left(\mathrm{~cm}^{-1}\right): 1503.41$
TABLE 1: Synthesized 1-aroyl-3-aryl thiourea derivatives.

\begin{tabular}{|c|c|c|c|}
\hline S. Number & Compound ID & $\mathrm{R}_{1}$ & $\mathrm{R}_{2}$ \\
\hline 1 & $\mathrm{Cl}$ & $2-\mathrm{Cl}$ & $4-\mathrm{Br}$ \\
\hline 2 & $\mathrm{C} 2$ & $4-\mathrm{Cl}$ & 3-OMe \\
\hline 3 & C3 & 4-OMe & 3-OMe \\
\hline 4 & $\mathrm{C} 4$ & 4-OMe & $2-\mathrm{Cl}$ \\
\hline 5 & C5 & $4-\mathrm{Cl}$ & $2-\mathrm{Cl}$ \\
\hline 6 & C6 & $4-\mathrm{Cl}$ & $4-\mathrm{F}$ \\
\hline 7 & C7 & $2-\mathrm{Cl}$ & 3-OMe \\
\hline 8 & $\mathrm{C} 8$ & 4-OMe & $4-\mathrm{Br}$ \\
\hline 9 & C9 & $2-\mathrm{Cl}$ & $4-\mathrm{Cl}$ \\
\hline 10 & $\mathrm{C} 10$ & $4-\mathrm{Cl}$ & $4-\mathrm{Cl}$ \\
\hline 11 & C11 & 4-OMe & $4-\mathrm{Cl}$ \\
\hline 12 & $\mathrm{C} 12$ & $2-\mathrm{Cl}$ & $2-\mathrm{F}$ \\
\hline 13 & $\mathrm{C} 13$ & $2-\mathrm{Cl}$ & 4-OMe \\
\hline 14 & $\mathrm{C} 14$ & $4-\mathrm{Cl}$ & 4-OMe \\
\hline 15 & $\mathrm{C} 15$ & 4-OMe & 4-OMe \\
\hline 16 & $\mathrm{C} 16$ & $4-\mathrm{Cl}$ & $2-\mathrm{F}$ \\
\hline 17 & $\mathrm{C} 17$ & 4-OMe & $2-\mathrm{F}$ \\
\hline 18 & $\mathrm{C} 18$ & $2-\mathrm{Cl}$ & $2-\mathrm{Cl}$ \\
\hline
\end{tabular}

$(\mathrm{C}=\mathrm{C}), 1659.63(\mathrm{C}=\mathrm{O}), 3517.92(\mathrm{~N}-\mathrm{H}), 1167.82(\mathrm{C}=\mathrm{S}), 1250.75$ $(\mathrm{C}-\mathrm{N}) .{ }^{1} \mathrm{H} \mathrm{NMR}\left(\mathrm{CDCl}_{3}\right) \delta: 12.69(1 \mathrm{H}, \mathrm{s}, \mathrm{NH}), 9.02(1 \mathrm{H}, \mathrm{s}$, $\mathrm{NH}$ ), 7.2-7.87 ( $\mathrm{m}, 4 \mathrm{H}$, aroyl ring), 6.81-7.02 ( $\mathrm{m}, 4 \mathrm{H}$, aryl ring), $3.83\left(3 \mathrm{H}, \mathrm{s}, \mathrm{OCH}_{3}\right), 3.89\left(3 \mathrm{H}, \mathrm{s}, \mathrm{OCH}_{3}\right)$, DART-MS m/e (\%): 315.12. Elemental analysis $\mathrm{C}_{16} \mathrm{H}_{16} \mathrm{~N}_{2} \mathrm{O}_{3} \mathrm{~S}(\mathrm{MW}=316.38)$ in wt $\%$ calc. $\mathrm{C}=60.74, \mathrm{H}=5.09, \mathrm{~N}=8.85, \mathrm{O}=15.17, \mathrm{~S}=$ 10.14 and found to be $\mathrm{C}=61.78, \mathrm{H}=5.52, \mathrm{~N}=8.79, \mathrm{O}=$ $15.37, \mathrm{~S}=8.43$.

1-(4-Methoxybenzoyl)-3-(2-chlorophenyl) Thiourea (C4). Yield $68 \%, R_{f}=0.32$, M.P. $140-145^{\circ} \mathrm{C}$, IR $(\mathrm{KBr}) \nu\left(\mathrm{cm}^{-1}\right): 1546.8$ $(\mathrm{C}=\mathrm{C}), 1673.81(\mathrm{C}=\mathrm{O}), 3257.55(\mathrm{~N}-\mathrm{H}), 1178.43(\mathrm{C}=\mathrm{S}), 1164.92$ $(\mathrm{C}-\mathrm{N}), 746.4(\mathrm{C}-\mathrm{Cl}) .{ }^{1} \mathrm{H}$ NMR $\left(\mathrm{CDCl}_{3}\right) \delta: 12.80(1 \mathrm{H}, \mathrm{s}$, $\mathrm{NH}), 9.11(1 \mathrm{H}, \mathrm{s}, \mathrm{NH}), 7.19-7.90$ (m, 4H, aroyl ring), 6.997.36 (m, 4H, aryl ring), $3.89\left(3 \mathrm{H}, \mathrm{s}, \mathrm{OCH}_{3}\right)$. DART-MS m/e (\%): 321.09. Elemental analysis for $\mathrm{C}_{15} \mathrm{H}_{13} \mathrm{~N}_{2} \mathrm{O}_{2} \mathrm{SCl}$ (MW = 320.79 ) in wt $\%$ calc. $\mathrm{C}=56.16, \mathrm{H}=4.08, \mathrm{~N}=8.73, \mathrm{O}=9.97$, $\mathrm{S}=9.99$ and found to be $\mathrm{C}=57.19, \mathrm{H}=4.47, \mathrm{~N}=8.75, \mathrm{O}=$ $10.13, \mathrm{~S}=9.81$.

1-(4-Chlorobenzoyl)-3-(2-chlorophenyl) Thiourea (C5). Yield $56 \%, R_{f}=0.45$, M.P. $162-165^{\circ} \mathrm{C}$, IR $(\mathrm{KBr}) v\left(\mathrm{~cm}^{-1}\right): 1537.16$ $(\mathrm{C}=\mathrm{C}), 1693.38(\mathrm{C}=\mathrm{O}), 3331.8(\mathrm{~N}-\mathrm{H}), 1094.53(\mathrm{C}=\mathrm{S}), 1014.49$ $(\mathrm{C}-\mathrm{N}), 687.58(\mathrm{C}-\mathrm{Cl}) .{ }^{1} \mathrm{H}$ NMR $\left(\mathrm{CDCl}_{3}\right) \delta: 12.65(1 \mathrm{H}, \mathrm{s}$, $\mathrm{NH}), 9.13$ (1H, s, NH), 7.53-7.88 (m, 4H, aroyl ring), 7.21-7.47 (m, $4 \mathrm{H}$, aryl ring). DART-MS m/e (\%): 325.048. Elemental analysis for $\mathrm{C}_{14} \mathrm{H}_{10} \mathrm{~N}_{2} \mathrm{OSCl}_{2}(\mathrm{MW}=325.22)$ in wt $\%$ calc. $\mathrm{C}=$ $51.71, \mathrm{H}=3.09, \mathrm{~N}=8.61, \mathrm{O}=4.91, \mathrm{~S}=9.85$ and found to be $\mathrm{C}=52.04, \mathrm{H}=3.29, \mathrm{~N}=8.18, \mathrm{O}=5.31, \mathrm{~S}=9.28$. 
1-(4-Chlorobenzoyl)-3-(4-fluorophenyl) Thiourea (C6). Yield $71 \%, R_{f}=0.50$, M.P. $155-158^{\circ} \mathrm{C}$, IR $(\mathrm{KBr}) v\left(\mathrm{~cm}^{-1}\right): 1537.16$ $(\mathrm{C}=\mathrm{C}), 1666.38(\mathrm{C}=\mathrm{O}), 3335.37(\mathrm{~N}-\mathrm{H}), 1157.21(\mathrm{C}=\mathrm{S}), 1157.21$ $(\mathrm{C}-\mathrm{N}), 826.44(\mathrm{C}-\mathrm{Cl}) .{ }^{1} \mathrm{H} \mathrm{NMR}\left(\mathrm{CDCl}_{3}\right) \delta: 12.43(1 \mathrm{H}, \mathrm{s}, \mathrm{NH})$, $9.05(1 \mathrm{H}, \mathrm{s}, \mathrm{NH}), 7.65-7.86(\mathrm{~m}, 4 \mathrm{H}$, aroyl ring), 7.09-7.26 (m, $4 \mathrm{H}$, aryl ring). DART-MS m/e (\%): 309.06. Elemental analysis for $\mathrm{C}_{14} \mathrm{H}_{10} \mathrm{~N}_{2} \mathrm{OSClF}(\mathrm{MW}=308.76)$ in wt $\%$ calc. $\mathrm{C}=54.46$, $\mathrm{H}=3.26, \mathrm{~N}=9.07, \mathrm{~S}=10.39$ and found to be $\mathrm{C}=54.59, \mathrm{H}=$ $3.41, \mathrm{~N}=9.13, \mathrm{~S}=10.43$.

1-(2-Chlorobenzoyl)-3-(3-methoxyphenyl) Thiourea (C7). Yield $48 \%, R_{f}=0.28$, M.P. $110-113^{\circ} \mathrm{C}$, IR $(\mathrm{KBr}) \nu\left(\mathrm{cm}^{-1}\right): 1503.41$ $(\mathrm{C}=\mathrm{C}), 1666.38(\mathrm{C}=\mathrm{O}), 3326.01(\mathrm{~N}-\mathrm{H}), 1148.53(\mathrm{C}=\mathrm{S}), 1036.67$ $(\mathrm{C}-\mathrm{N}), 742.54(\mathrm{C}-\mathrm{Cl}) .{ }^{1} \mathrm{H} \mathrm{NMR}\left(\mathrm{CDCl}_{3}\right) \delta: 12.68(1 \mathrm{H}, \mathrm{s}, \mathrm{NH})$, $9.22(1 \mathrm{H}, \mathrm{s}, \mathrm{NH}), 7.43-7.78(\mathrm{~m}, 4 \mathrm{H}$, aroyl ring), 6.83-7.22 (m, $4 \mathrm{H}$, aryl ring), 3.84 (3H, s, $\mathrm{OCH}_{3}$ ). DART-MS m/e (\%): 321. Elemental analysis for $\mathrm{C}_{15} \mathrm{H}_{13} \mathrm{~N}_{2} \mathrm{O}_{2} \mathrm{SCl}(\mathrm{MW}=320.79)$ in wt $\%$ calc. $\mathrm{C}=56.16, \mathrm{H}=4.09, \mathrm{~N}=8.73, \mathrm{~S}=9.99$ and found to be $\mathrm{C}=56.41, \mathrm{H}=4.43, \mathrm{~N}=8.8, \mathrm{~S}=9.97$.

1-(4-Methoxybenzoyl)-3-(4-bromophenyl) Thiourea (C8). Yield $82 \%, R_{f}=0.31$, M.P. $124-128^{\circ} \mathrm{C}$, IR $(\mathrm{KBr}) \nu\left(\mathrm{cm}^{-1}\right): 1503.41$ $(\mathrm{C}=\mathrm{C}), 1666.38(\mathrm{C}=\mathrm{O}), 3376.16(\mathrm{~N}-\mathrm{H}), 1073.31(\mathrm{C}=\mathrm{S}), 1024.13$ $(\mathrm{C}-\mathrm{N}) .{ }^{1} \mathrm{H}$ NMR $\left(\mathrm{CDCl}_{3}\right) \delta: 12.56(1 \mathrm{H}, \mathrm{s}, \mathrm{NH}), 9.01(1 \mathrm{H}$, $\mathrm{s}, \mathrm{NH}), 7.62-7.88(\mathrm{~m}, 4 \mathrm{H}$, aroyl ring), 7.0-7.51 (m, $4 \mathrm{H}$, aryl ring), $3.9\left(3 \mathrm{H}, \mathrm{s}, \mathrm{OCH}_{3}\right)$. DART-MS m/e (\%): 365. Elemental analysis for $\mathrm{C}_{15} \mathrm{H}_{13} \mathrm{~N}_{2} \mathrm{O}_{2} \mathrm{SBr}(\mathrm{MW}=365.25)$ in $\mathrm{wt} \%$ calc. $\mathrm{C}=49.33, \mathrm{H}=3.58, \mathrm{~N}=7.67, \mathrm{~S}=8.78$ and found to be $\mathrm{C}=$ 49.58, $\mathrm{H}=3.55, \mathrm{~N}=7.82, \mathrm{~S}=8.96$.

1-(2-Chlorobenzoyl)-3-(4-chlorophenyl) Thiourea (C9). Yield $64 \%, R_{f}=0.38$, M.P. $188-190^{\circ} \mathrm{C}$, IR $(\mathrm{KBr}) \nu\left(\mathrm{cm}^{-1}\right): 1504.37$ $(\mathrm{C}=\mathrm{C}), 1666.38(\mathrm{C}=\mathrm{O}), 3267.19(\mathrm{~N}-\mathrm{H}), 1155.28(\mathrm{C}=\mathrm{S}), 1155.28$ $(\mathrm{C}-\mathrm{N}), 831.26(\mathrm{C}-\mathrm{Cl}) .{ }^{1} \mathrm{H}$ NMR $\left(\mathrm{CDCl}_{3}\right) \delta: 12.93(1 \mathrm{H}, \mathrm{s}, \mathrm{NH})$, $9.15(1 \mathrm{H}, \mathrm{s}, \mathrm{NH}), 7.63-7.74(\mathrm{~m}, 4 \mathrm{H}$, aroyl ring), 6.73-7.26 (m, $4 \mathrm{H}$, aryl ring). DART-MS m/e (\%): 325.66. Elemental analysis for $\mathrm{C}_{14} \mathrm{H}_{10} \mathrm{~N}_{2} \mathrm{OSCl}_{2}(\mathrm{MW}=325.22)$ in wt\% calc. $\mathrm{C}=51.70$, $\mathrm{H}=3.09, \mathrm{~N}=8.61, \mathrm{~S}=9.85$ and found to be $\mathrm{C}=51.92, \mathrm{H}=$ $3.20, \mathrm{~N}=9.04, \mathrm{~S}=9.17$.

1-(4-Chlorobenzoyl)-3-(4-chlorophenyl) Thiourea (C10). Yield $57 \%, R_{f}=0.2$, M.P. $183-187^{\circ} \mathrm{C}$, IR $(\mathrm{KBr}) v\left(\mathrm{~cm}^{-1}\right)$ : $1537.16(\mathrm{C}=\mathrm{C}), 1686.63(\mathrm{C}=\mathrm{O}), 3381.56(\mathrm{~N}-\mathrm{H}), 1095.49(\mathrm{C}=\mathrm{S})$, $1095.49(\mathrm{C}-\mathrm{N}), 820.65(\mathrm{C}-\mathrm{Cl}) .{ }^{1} \mathrm{H} \mathrm{NMR}\left(\mathrm{CDCl}_{3}\right) \delta: 12.64$ $(1 \mathrm{H}, \mathrm{s}, \mathrm{NH}), 9.03(1 \mathrm{H}, \mathrm{s}, \mathrm{NH}), 7.66-7.83$ (m, 4H, aroyl ring), 7.26-7.52 ( $\mathrm{m}, 4 \mathrm{H}$, aryl ring). DART-MS m/e (\%): 325.86. Elemental analysis for $\mathrm{C}_{14} \mathrm{H}_{10} \mathrm{~N}_{2} \mathrm{OSCl}_{2}(\mathrm{MW}=325.22)$ in wt\% calc. $\mathrm{C}=51.70, \mathrm{H}=3.09, \mathrm{~N}=8.61, \mathrm{~S}=9.85$ and found to be $\mathrm{C}=51.93, \mathrm{H}=2.96, \mathrm{~N}=8.65, \mathrm{~S}=9.49$.

1-(4-Methoxybenzoyl)-3-(4-chlorophenyl) Thiourea (C11). Yield $65 \%, R_{f}=0.29$, M.P. $182-187^{\circ} \mathrm{C}$, IR $(\mathrm{KBr}) \nu\left(\mathrm{cm}^{-1}\right): 1537.16$ $(\mathrm{C}=\mathrm{C}), 1666.38(\mathrm{C}=\mathrm{O}), 3388.7(\mathrm{~N}-\mathrm{H}), 1174.57(\mathrm{C}=\mathrm{S}), 1250.75$ $(\mathrm{C}-\mathrm{N}), 822.58(\mathrm{C}-\mathrm{Cl}) .{ }^{1} \mathrm{H} \mathrm{NMR}\left(\mathrm{CDCl}_{3}\right) \delta: 12.69(1 \mathrm{H}, \mathrm{s}, \mathrm{NH})$, $9.05(1 \mathrm{H}, \mathrm{s}, \mathrm{NH}), 7.26-7.87(\mathrm{~m}, 4 \mathrm{H}$, aroyl ring), 6.9-7.36 (m, $4 \mathrm{H}$, aryl ring), 3.9 ( $\left.3 \mathrm{H}, \mathrm{s}, \mathrm{OCH}_{3}\right)$. DART-MS m/e (\%): 321.09. Elemental analysis for $\mathrm{C}_{15} \mathrm{H}_{13} \mathrm{~N}_{2} \mathrm{O}_{2} \mathrm{SCl}(\mathrm{MW}=320.79)$ in wt $\%$ calc. $\mathrm{C}=56.16, \mathrm{H}=4.08, \mathrm{~N}=8.73, \mathrm{~S}=9.99$ and found to be $\mathrm{C}=56.53, \mathrm{H}=4.33, \mathrm{~N}=8.98, \mathrm{~S}=9.97$.
1-(2-Chlorobenzoyl)-3-(2-fluorophenyl) Thiourea (C12). Yield $76 \%, R_{f}=0.44$, M.P. $162-170^{\circ} \mathrm{C}$, IR $(\mathrm{KBr}) \nu\left(\mathrm{cm}^{-1}\right): 1536.2$ $(\mathrm{C}=\mathrm{C}), 1686.63(\mathrm{C}=\mathrm{O}), 3327.65(\mathrm{~N}-\mathrm{H}), 1202.53(\mathrm{C}=\mathrm{S}), 1163$ $(\mathrm{C}-\mathrm{N}), 753.15(\mathrm{C}-\mathrm{Cl}) .{ }^{1} \mathrm{H} \mathrm{NMR}\left(\mathrm{CDCl}_{3}\right) \delta: 12.46(1 \mathrm{H}, \mathrm{s}, \mathrm{NH})$, $9.37(1 \mathrm{H}, \mathrm{s}, \mathrm{NH}), 7.43-7.78(\mathrm{~m}, 4 \mathrm{H}$, aroyl ring), 7.15-7.20 (m, $4 \mathrm{H}$, aryl ring). DART-MS m/e (\%): 309.08. Elemental analysis for $\mathrm{C}_{14} \mathrm{H}_{10} \mathrm{~N}_{2} \mathrm{OSClF}(\mathrm{MW}=308.76)$ in wt $\%$ calc. $\mathrm{C}=54.46$, $\mathrm{H}=3.26, \mathrm{~N}=9.07, \mathrm{~S}=10.39$ and found to be $\mathrm{C}=54.7, \mathrm{H}=$ $3.05, \mathrm{~N}=9.16, \mathrm{~S}=10.42$.

1-(2-Chlorobenzoyl)-3-(4-methoxyphenyl) Thiourea (C13). Yield $53 \%, R_{f}=0.24$, M.P. $156-162^{\circ} \mathrm{C}$, IR $(\mathrm{KBr}) \nu\left(\mathrm{cm}^{-1}\right): 1537.16$ $(\mathrm{C}=\mathrm{C}), 1666.38(\mathrm{C}=\mathrm{O}), 3251.11(\mathrm{~N}-\mathrm{H}), 1159.14(\mathrm{C}=\mathrm{S}), 1026.06$ $(\mathrm{C}-\mathrm{N}), 744.47(\mathrm{C}-\mathrm{Cl}) .{ }^{1} \mathrm{H}$ NMR $\left(\mathrm{CDCl}_{3}\right) \delta: 12.18(1 \mathrm{H}, \mathrm{s}, \mathrm{NH})$, $9.28(1 \mathrm{H}, \mathrm{s}, \mathrm{NH}), 7.43-7.59(\mathrm{~m}, 4 \mathrm{H}$, aroyl ring), 6.93-7.39 (m, $4 \mathrm{H}$, aryl ring), 3.83 ( $3 \mathrm{H}, \mathrm{s}, \mathrm{OCH}_{3}$ ). DART-MS m/e (\%): 321. Elemental analysis for $\mathrm{C}_{15} \mathrm{H}_{13} \mathrm{~N}_{2} \mathrm{O}_{2} \mathrm{SCl}(\mathrm{MW}=320.79)$ in wt $\%$ calc. $\mathrm{C}=56.16, \mathrm{H}=4.08, \mathrm{~N}=8.73, \mathrm{~S}=9.99$ and found to be $\mathrm{C}=56.38, \mathrm{H}=4.27, \mathrm{~N}=9.07, \mathrm{~S}=9.77$.

1-(4-Chlorobenzoyl)-3-(4-methoxyphenyl) Thiourea (C14).Yield $91 \%, R_{f}=0.41$, M.P. $122-130^{\circ} \mathrm{C}$, IR $(\mathrm{KBr}) \nu\left(\mathrm{cm}^{-1}\right): 1597.91$ $(\mathrm{C}=\mathrm{C}), 1693.38(\mathrm{C}=\mathrm{O}), 3360.82(\mathrm{~N}-\mathrm{H}), 1094.53(\mathrm{C}=\mathrm{S}), 1241.11$ $(\mathrm{C}-\mathrm{N}), 743.51(\mathrm{C}-\mathrm{Cl}) .{ }^{1} \mathrm{H} \mathrm{NMR}\left(\mathrm{CDCl}_{3}\right) \delta: 12.33(1 \mathrm{H}, \mathrm{s}, \mathrm{NH})$, $9.10(1 \mathrm{H}, \mathrm{s}, \mathrm{NH}), 7.54-7.85(\mathrm{~m}, 4 \mathrm{H}$, aroyl ring), 6.93-7.50 (m, $4 \mathrm{H}$, aryl ring), 3.83 (3H, s, $\mathrm{OCH}_{3}$ ). DART-MS m/e (\%): 321.10 . Elemental analysis for $\mathrm{C}_{15} \mathrm{H}_{13} \mathrm{~N}_{2} \mathrm{O}_{2} \mathrm{SCl}(\mathrm{MW}=320.79)$ in wt $\%$ calc. $\mathrm{C}=56.16, \mathrm{H}=4.08, \mathrm{~N}=8.73, \mathrm{~S}=9.99$ and found to be $\mathrm{C}=56.29, \mathrm{H}=4.24, \mathrm{~N}=9.02, \mathrm{~S}=9.98$.

1-(4-Methoxybenzoyl)-3-(4-methoxyphenyl) Thiourea (C15). Yield $62 \%, R_{f}=0.30$, M.P. $122-128^{\circ} \mathrm{C}$, IR $(\mathrm{KBr}) \nu\left(\mathrm{cm}^{-1}\right)$ : $1504.37(\mathrm{C}=\mathrm{C}), 1666.38(\mathrm{C}=\mathrm{O}), 3287.44(\mathrm{~N}-\mathrm{H}), 1174.57(\mathrm{C}=\mathrm{S})$, $1245.93(\mathrm{C}-\mathrm{N}) .{ }^{1} \mathrm{H}$ NMR $\left(\mathrm{CDCl}_{3}\right) \delta: 12.48(1 \mathrm{H}, \mathrm{s}, \mathrm{NH}), 9.07$ $(1 \mathrm{H}, \mathrm{s}, \mathrm{NH}), 7.54-7.87(\mathrm{~m}, 4 \mathrm{H}$, aroyl ring), 6.94-7.01 (m, $4 \mathrm{H}$, aryl ring), $3.82\left(3 \mathrm{H}, \mathrm{s}, \mathrm{OCH}_{3}\right), 3.88\left(3 \mathrm{H}, \mathrm{s}, \mathrm{OCH}_{3}\right)$. DART-MS $\mathrm{m} / \mathrm{e}(\%): 317.15$. Elemental analysis for $\mathrm{C}_{16} \mathrm{H}_{16} \mathrm{~N}_{2} \mathrm{O}_{3} \mathrm{~S}(\mathrm{MW}=$ 316.38 ) in wt $\%$ calc. $\mathrm{C}=60.74, \mathrm{H}=5.09, \mathrm{~N}=8.85, \mathrm{~S}=10.14$ and found to be $\mathrm{C}=61.10, \mathrm{H}=5.45, \mathrm{~N}=9.36, \mathrm{~S}=9.36$.

1-(4-Chlorobenzoyl)-3-(2-fluorophenyl) Thiourea (C16). Yield $67 \%, R_{f}=0.48$, M.P. $148-152^{\circ} \mathrm{C}$, IR $(\mathrm{KBr}) \nu\left(\mathrm{cm}^{-1}\right): 1537.16$ $(\mathrm{C}=\mathrm{C}), 1666.38(\mathrm{C}=\mathrm{O}), 3296.85(\mathrm{~N}-\mathrm{H}), 1201.57(\mathrm{C}=\mathrm{S}), 1239.18$ $(\mathrm{C}-\mathrm{N}), 747.36(\mathrm{C}-\mathrm{Cl}) .{ }^{1} \mathrm{H} \mathrm{NMR}\left(\mathrm{CDCl}_{3}\right) \delta: 12.60(1 \mathrm{H}, \mathrm{s}, \mathrm{NH})$, $9.15(1 \mathrm{H}, \mathrm{s}, \mathrm{NH}), 7.51-7.87$ (m, 4H, aroyl ring), 7.15-7.2 (m, 4H, aryl ring). DART-MS m/e (\%): 309.7. Elemental analysis for $\mathrm{C}_{14} \mathrm{H}_{10} \mathrm{~N}_{2}$ OSClF (MW = 308.76) in wt\% calc. $\mathrm{C}=54.46$, $\mathrm{H}=3.26, \mathrm{~N}=9.07, \mathrm{~S}=10.39$ and found to be $\mathrm{C}=54.60, \mathrm{H}=$ $3.07, \mathrm{~N}=9.20, \mathrm{~S}=10.41$.

1-(4-Methoxybenzoyl)-3-(2-fluorophenyl) Thiourea (C17). Yield $59 \%, R_{f}=0.30$, M.P. $122-126^{\circ} \mathrm{C}$, IR (KBr) $\nu\left(\mathrm{cm}^{-1}\right): 1504.38$ $(\mathrm{C}=\mathrm{C}), 1666.38(\mathrm{C}=\mathrm{O}), 3294.19(\mathrm{~N}-\mathrm{H}), 1194.82(\mathrm{C}=\mathrm{S}), 1028.95$ $(\mathrm{C}-\mathrm{N}) .{ }^{1} \mathrm{H} \mathrm{NMR}\left(\mathrm{CDCl}_{3}\right) \delta: 12.76(1 \mathrm{H}, \mathrm{s}, \mathrm{NH}), 9.11(1 \mathrm{H}, \mathrm{s}$, $\mathrm{NH}), 7.02-7.89$ (m, 4H, aroyl ring), 6.99-7.15 (m, 4H, aryl ring), $3.89\left(3 \mathrm{H}, \mathrm{s}, \mathrm{OCH}_{3}\right)$. DART-MS m/e (\%): 305.12. Elemental analysis for $\mathrm{C}_{15} \mathrm{H}_{13} \mathrm{~N}_{2} \mathrm{O}_{2} \mathrm{SF}(\mathrm{MW}=304.34)$ in wt $\%$ calc. $\mathrm{C}=59.20, \mathrm{H}=4.30, \mathrm{~N}=9.20, \mathrm{~S}=10.55$ and found to be $\mathrm{C}=59.38, \mathrm{H}=4.30, \mathrm{~N}=9.60, \mathrm{~S}=10.83$. 
TABLE 2: Zone of inhibition for samples, standard, and control.

\begin{tabular}{|c|c|c|c|c|}
\hline \multirow[b]{2}{*}{ Comp. ID } & \multicolumn{2}{|c|}{ Escherichia coli } & \multicolumn{2}{|c|}{ Staphylococcus aureus } \\
\hline & $\begin{array}{l}\text { Zone of inhibition } \\
\text { (in } \mathrm{mm} \text { ) }\end{array}$ & $\begin{array}{c}\text { Relative inhibition } \\
\text { zone diameter (in \%) }\end{array}$ & $\begin{array}{c}\text { Zone of inhibition } \\
\text { (in } \mathrm{mm} \text { ) }\end{array}$ & $\begin{array}{c}\text { Relative inhibition zone } \\
\text { diameter (in \%) }\end{array}$ \\
\hline $\mathrm{C} 1$ & 11.7 & 72.7 & 10.9 & 71.2 \\
\hline $\mathrm{C} 2$ & 7.2 & 44.7 & 6.4 & 41.8 \\
\hline C3 & 10.6 & 65.8 & 9.0 & 58.8 \\
\hline $\mathrm{C} 4$ & 9.1 & 56.5 & 8.4 & 54.9 \\
\hline C5 & 14.2 & 88.2 & 13.1 & 85.6 \\
\hline C6 & 12.1 & 75.2 & 11.6 & 75.8 \\
\hline C7 & 8.4 & 52.2 & 7.9 & 51.6 \\
\hline $\mathrm{C} 8$ & 7.2 & 44.7 & 7.0 & 45.8 \\
\hline C9 & 11.5 & 71.4 & 10.4 & 68.0 \\
\hline $\mathrm{C} 10$ & 12.8 & 79.5 & 10.9 & 71.2 \\
\hline C11 & 7.4 & 46.0 & 7.9 & 51.6 \\
\hline $\mathrm{C} 12$ & 12.3 & 76.4 & 12.0 & 78.4 \\
\hline $\mathrm{C} 13$ & 6.4 & 39.8 & 6.0 & 39.2 \\
\hline $\mathrm{C} 14$ & 5.2 & 32.3 & 4.0 & 26.1 \\
\hline $\mathrm{C} 15$ & 5.7 & 35.4 & 4.2 & 27.5 \\
\hline $\mathrm{C} 16$ & 13.8 & 85.7 & 13.0 & 85.0 \\
\hline $\mathrm{C} 17$ & 10.6 & 65.8 & 9.4 & 61.4 \\
\hline $\mathrm{C} 18$ & 14.4 & 89.4 & 12.6 & 82.4 \\
\hline Standard & 16.1 & - & 15.3 & - \\
\hline Control & \multicolumn{2}{|c|}{ Nil } & \multicolumn{2}{|c|}{ Nil } \\
\hline
\end{tabular}

Standard: Sodium Penicillin G, Control: control (Solvent-DMSO).

1-(2-Chlorobenzoyl)-3-(2-chlorophenyl) Thiourea (C18). Yield $73 \%, R_{f}=0.35$, M.P. $170-174^{\circ} \mathrm{C}$, IR $(\mathrm{KBr}) v\left(\mathrm{~cm}^{-1}\right): 1537.16$ $(\mathrm{C}=\mathrm{C}), 1686.63(\mathrm{C}=\mathrm{O}), 3325.30(\mathrm{~N}-\mathrm{H}), 1162.03(\mathrm{C}=\mathrm{S}), 1162.03$ $(\mathrm{C}-\mathrm{N}), 756.04(\mathrm{C}-\mathrm{Cl}) .{ }^{1} \mathrm{H} \mathrm{NMR}\left(\mathrm{CDCl}_{3}\right) \delta: 12.55(1 \mathrm{H}, \mathrm{s}, \mathrm{NH})$, $9.39(1 \mathrm{H}, \mathrm{s}, \mathrm{NH}), 7.43-7.83(\mathrm{~m}, 4 \mathrm{H}$, aroyl ring), 7.21-7.40 (m, $4 \mathrm{H}$, aryl ring). DART-MS m/e (\%): 325.04. Elemental analysis for $\mathrm{C}_{14} \mathrm{H}_{10} \mathrm{~N}_{2} \mathrm{OSCl}_{2}(\mathrm{MW}=325.22)$ in wt\% calc. $\mathrm{C}=59.20$, $\mathrm{H}=4.30, \mathrm{~N}=9.20, \mathrm{~S}=10.55$ and found to be $\mathrm{C}=59.38, \mathrm{H}=$ $4.30, \mathrm{~N}=9.60, \mathrm{~S}=10.83$.

\section{Results and Discussion}

3.1. Chemistry. Synthesis of some 1-aroyl-3-aryl thioureas (C1-C18) was carried out by treating substituted benzoyl chloride with an equimolar quantity of potassium thiocyanate in acetone followed by reaction with an equimolar amount of substituted anilines to furnish the 1-aroyl-3-aryl thiourea derivatives.

The structures were confirmed by spectroscopic data and elemental analyses. FT-IR spectrum of synthesized thiourea derivatives exhibited identical trend of stretching frequency modes. Presence of peaks in the regions $3200-3250 \mathrm{~cm}^{-1}$ and $3320-3500 \mathrm{~cm}^{-1}$ indicates the existence of $\mathrm{NH}$ moiety and that of peaks in the regions $1665-1695 \mathrm{~cm}^{-1}$ and $1050-1200$ indicates $\mathrm{C}=\mathrm{O}$ and $\mathrm{C}=\mathrm{S}$ moieties, respectively, in all the compounds. NMR spectra revealed the presence of characteristic broad singlets for $\mathrm{HN}(1)$ and $\mathrm{HN}(3)$ at 9 and $12 \mathrm{ppm}$, respectively. Mass spectra of all the compounds displayed molecular ion peaks. Elemental analysis data are also in line with the theoretical values.

3.2. Antibacterial Activity. The synthesized compounds were assayed for their antibacterial activity against Staphylococcus aureus 6538 (gram-positive bacteria) and Escherichia coli 8739 (gram-negative bacteria) using paper disc diffusion method with Mueller-Hinton agar medium. Sodium Penicillin $\mathrm{G}$ was used as the reference antibacterial agent.

All the synthesized compounds inhibited the growth of bacteria with zone of inhibition ranging between 4.2 and $13.1 \mathrm{~mm}$ and 5.2 and $14.4 \mathrm{~mm}$ for gram-positive bacteria and gram-negative bacteria, respectively. These compounds were found to be more active against gram-negative bacteria as compared to gram-positive bacteria. The antibacterial activity of all the compounds observed against both microorganisms is shown in Table 2.

3.2.1. Staphylococcus aureus (Gram Positive). Amongst all these compounds, C5 (85.6\%) substituted with chloro group on para position of aroyl ring and fluoro group on ortho position of aryl ring exhibits maximum percentage of relative zone of inhibition and C12 (78.4\%), C16 (85\%), and C18 (82.4) also displayed significant antibacterial activity, while $\mathrm{C} 14$ (27.9\%) containing fluoro and methoxy groups on para positions of aroyl and aryl ring, respectively, displayed the least 
percentage of relative zone of inhibition as compared with the standard drug. Following is the antibacterial efficacy of synthesized compounds against $S$. aureus:

$$
\begin{aligned}
& \mathrm{C} 5>\mathrm{C} 16>\mathrm{C} 18>\mathrm{C} 12>\mathrm{C} 6>\mathrm{C} 1>\mathrm{C} 10>\mathrm{C} 9>\mathrm{C} 17> \\
& \mathrm{C} 3>\mathrm{C} 4>\mathrm{C} 7>\mathrm{C} 11>\mathrm{C} 8>\mathrm{C} 2>\mathrm{C} 13>\mathrm{C} 15>\mathrm{C} 14 .
\end{aligned}
$$

3.2.2. E. coli (Gram Negative). Amongst all these compounds, C18 (89.4\%) carrying chloro groups on ortho positions of both aroyl and aryl rings displayed maximum antibacterial activity against E. coli and C5 (88.2\%), C16 (85.7\%), C10 (79.5\%), and C12 (76.4\%) exhibited significant percentage of relative zone of inhibition while $\mathrm{C} 14$ (32.3\%) substituted with fluoro and methoxy groups on para positions of aroyl and aryl ring, respectively, displayed the least percentage of relative zone of inhibition as compared with the standard drug. Following is the antibacterial efficacy of synthesized compounds against E. coli:

$$
\begin{aligned}
& \mathrm{C} 18>\mathrm{C} 5>\mathrm{C} 16>\mathrm{C} 10>\mathrm{C} 12>\mathrm{C} 6>\mathrm{C} 1>\mathrm{C} 9>\mathrm{C} 3> \\
& \mathrm{C} 17>\mathrm{C} 4>\mathrm{C} 7>\mathrm{C} 11>\mathrm{C} 2>\mathrm{C} 8>\mathrm{C} 13>\mathrm{C} 15>\mathrm{C} 14 .
\end{aligned}
$$

The synthesized compounds exhibited potent inhibitory activity against the two strains compared to standard drugs at the tested concentrations. Compounds containing chloro group at ortho position on aryl ring and chloro at para and ortho positions of aroyl ring, respectively, (C5 and C18) prove to be the active against both gram-positive and gramnegative strains, while C14 (32.3\%) substituted with fluoro and methoxy groups on para positions of aroyl and aryl ring, respectively, displayed the least percentage of relative zone of inhibition as compared with the standard drug against both $E$. coli and S. aureus. These observations suggested that presence of electron withdrawing group on aryl ring is crucial for antibacterial activity.

\section{Conclusion}

The title compounds were synthesized by treating substituted benzoyl chloride with an equimolar quantity of potassium thiocyanate in acetone followed by reaction with an equimolar amount of substituted anilines to furnish the 1-aroyl-3-aryl thiourea derivatives. The synthesized thiourea derivatives were found to be more active against gram-negative bacteria as compared to gram-positive bacteria. Among the synthesized compounds, C5 and C18 exhibited maximum relative zone of inhibition against gram-positive and gram-negative strains of bacteria, respectively, as compared with the standard. These observations suggested that presence of electron withdrawing group on aryl ring is crucial for antibacterial activity.

\section{Conflict of Interests}

The authors declare that there is no conflict of interests regarding the publication of this paper.

\section{Acknowledgments}

The authors acknowledge the Head, School of Pharmacy, and Honorable Vice-Chancellor, Devi Ahilya University, Indore, for providing facilities for the completion of this work.

\section{References}

[1] F. Karipcin, M. Atis, B. Sariboga, H. Celik, and M. Tas, "Structural, spectral, optical and antimicrobial properties of synthesized 1-benzoyl-3-furan-2-ylmethyl-thiourea," Journal of Molecular Structure, vol. 1048, pp. 69-77, 2013.

[2] J. M. Vega-Pérez, I. Periñán, M. Argandoña et al., "Isoprenylthiourea and urea derivatives as new farnesyl diphosphate analogues: synthesis and in vitro antimicrobial and cytotoxic activities," European Journal of Medicinal Chemistry, vol. 58, pp. 591612, 2012.

[3] A. Saeed, N. Saeed, R. Hummera, R. Sadaf, and A. Hameed, "Synthesis, characterization and antibacterial activity of some 1-aroyl-3-aryl thioureas," Chemistry, vol. 18, pp. 152-158, 2009.

[4] S. Saeed, N. Rashid, P. G. Jones, M. Ali, and R. Hussain, "Synthesis, characterization and biological evaluation of some thiourea derivatives bearing benzothiazole moiety as potential antimicrobial and anticancer agents," European Journal of Medicinal Chemistry, vol. 45, no. 4, pp. 1323-1331, 2010.

[5] K. Ekoue-Kovi, K. Yearick, D. P. Iwaniuk et al., "Synthesis and antimalarial activity of new 4-amino-7-chloroquinolyl amides, sulfonamides, ureas and thioureas," Bioorganic and Medicinal Chemistry, vol. 17, no. 1, pp. 270-283, 2009.

[6] A. Liav, S. K. Angala, P. J. Brennan, and M. Jackson, "N-DAldopentofuranosyl- $N^{\prime}$-[ $p$-(isoamyloxy)phenyl]-thiourea derivatives: potential anti-TB therapeutic agents," Bioorganic and Medicinal Chemistry Letters, vol. 18, no. 8, pp. 2649-2651, 2008.

[7] S. N. Manjula, N. M. Noolvi, K. V. Parihar et al., "Synthesis and antitumor activity of optically active thiourea and their 2-aminobenzothiazole derivatives: a novel class of anticancer agents," European Journal of Medicinal Chemistry, vol. 44, no. 7, pp. 2923-2929, 2009.

[8] S. B. Tsogoeva, M. J. Hateley, D. A. Yalalov, K. Meindl, C. Weckbecker, and K. Huthmacher, "Thiourea-based non-nucleoside inhibitors of HIV reverse transcriptase as bifunctional organocatalysts in the asymmetric Strecker synthesis," Bioorganic and Medicinal Chemistry, vol. 13, no. 19, pp. 5680-5685, 2005.

[9] J. Moeker, K. Teruya, S. Rossit et al., "Design and synthesis of thiourea compounds that inhibit transmembrane anchored carbonic anhydrases," Bioorganic and Medicinal Chemistry, vol. 20, no. 7, pp. 2392-2404, 2012.

[10] K. Bhandari, S. Srivastava, and G. Shankar, "Synthesis of tetrahydronaphthyl thioureas as potent appetite suppressants," Bioorganic and Medicinal Chemistry, vol. 12, no. 15, pp. 4189-4196, 2004.

[11] T. Maruyama, N. Seki, K. Onda et al., "Discovery of novel thiourea derivatives as potent and selective $\beta 3$-adrenergic receptor agonists," Bioorganic and Medicinal Chemistry, vol. 17, no. 15, pp. 5510-5519, 2009.

[12] J. Stefanska, D. Szulczyk, A. E. Koziol et al., "Disubstituted thiourea derivatives and their activity on CNS: synthesis and biological evaluation," European Journal of Medicinal Chemistry, vol. 55, pp. 205-213, 2012. 
[13] L. K. Soni, T. Narsinghani, and A. Sethi, "Anti-microbial benzimidazole derivatives: synthesis and in vitro biological evaluation," Medicinal Chemistry Research, vol. 21, pp. 4330-4334, 2012.

[14] R. A. Gupta, A. K. Gupta, L. K. Soni, and S. G. Kaskhedikar, "2-(pyrazin-2-yloxy)acetohydrazide analogs QSAR study: an insight into the structural basis of antimycobacterial activity," Chemical Biology and Drug Design, vol. 76, no. 5, pp. 441-450, 2010.

[15] A. Parate, L. K. Soni, and R. Malviya, "Synthesis and antibacterial evaluation of some substituted benzoxazole analogues," Der Pharmacia Sinica, vol. 4, no. 3, pp. 130-135, 2013. 

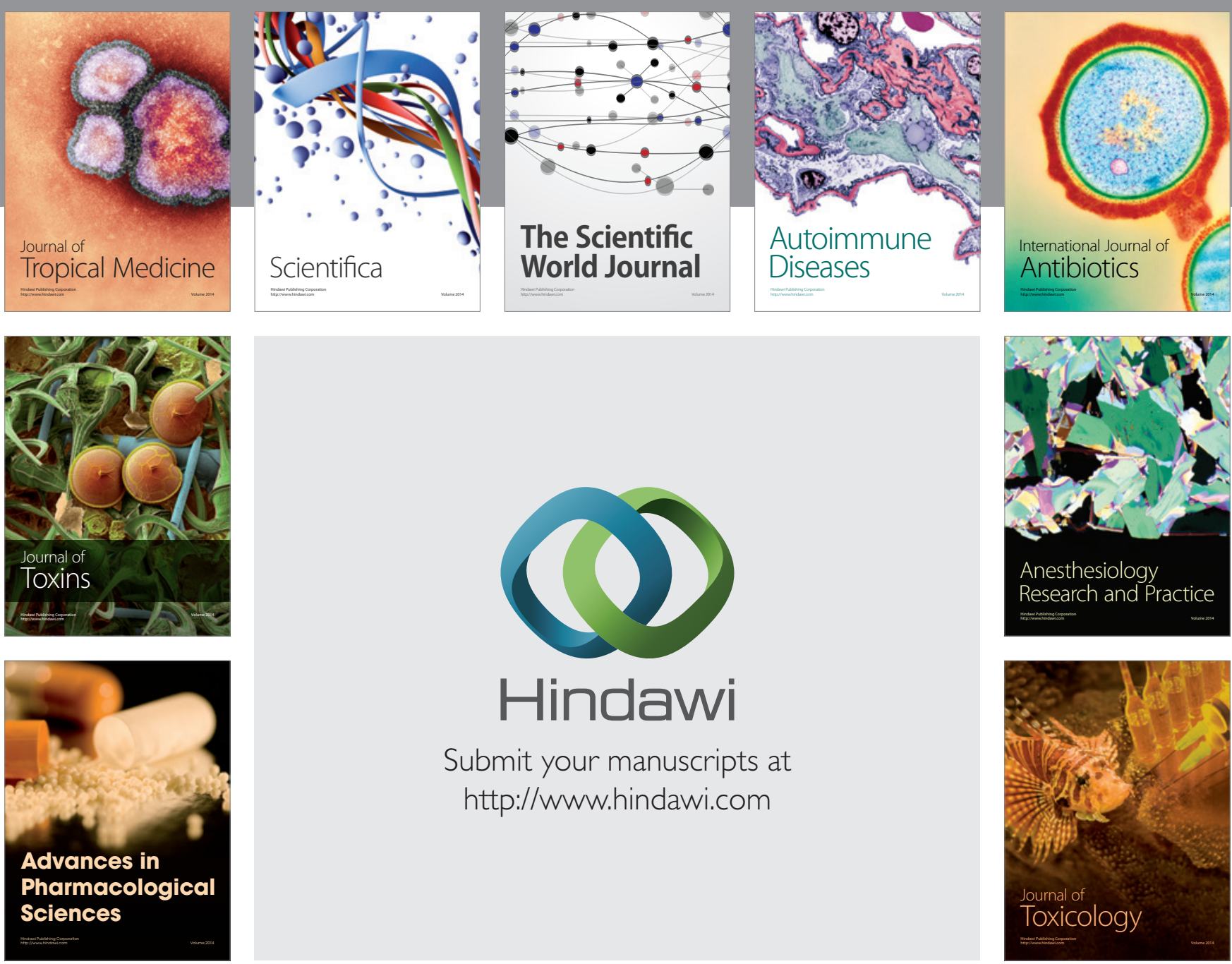

\section{Hindawi}

Submit your manuscripts at

http://www.hindawi.com
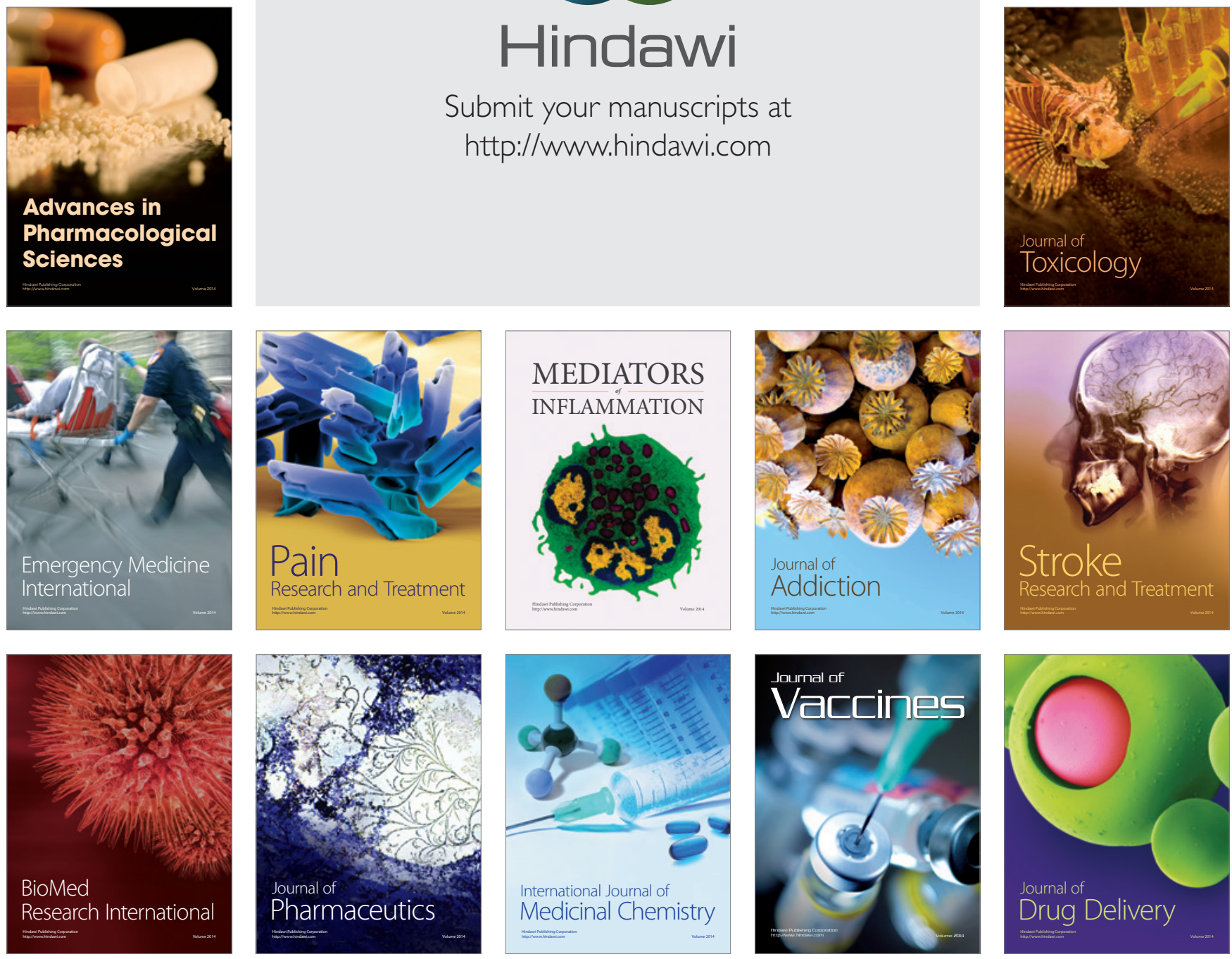